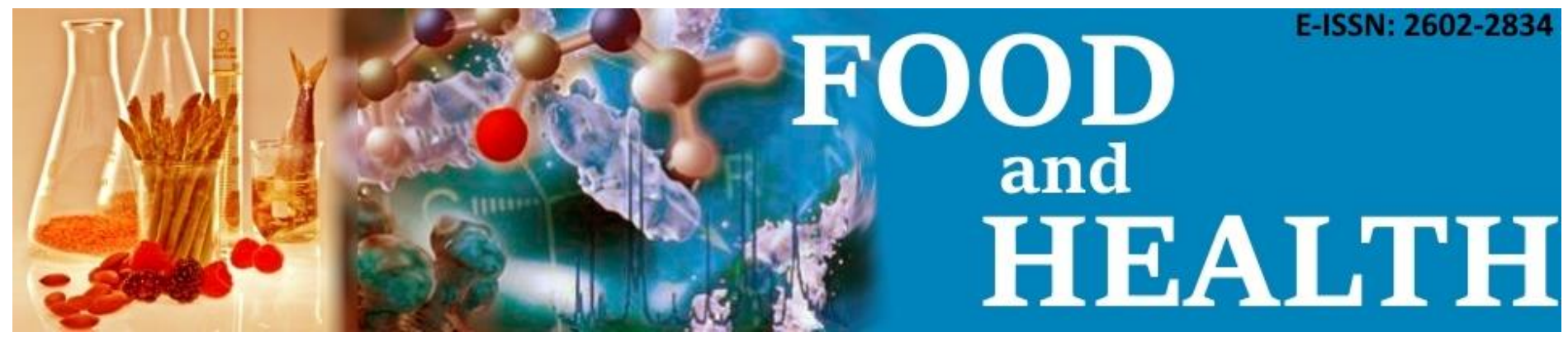

\title{
PHYSICOCHEMICAL, RHEOLOGICAL AND STRUCTURAL CHARACTERISTICS OF ALCOHOL PRECIPITATED FRACTION OF GUM TRAGACANTH
}

\author{
Abdullah Kurt i⿱D \\ Cite this article as: \\ Kurt, A. (2018). Physicochemical, rheological and structural characteristics of alcohol precipitated fraction of gum tragacanth. Food and Health, 4(3), \\ 183-193. DOI: $10.3153 / \mathrm{FH} 18019$
}

Bitlis Eren University, EngineeringArchitecture Faculty, Department of Food Engineering, 13000 Bitlis, Turkey

Submitted: 20.07 .2017

Accepted: 28.12.2017

Published online: 19.03.2018

Correspondence:

\section{Abdullah KURT}

E-mail: abdullahkurt48@gmail.com

\begin{abstract}
Obtaining specific polysaccharide of gum by separating other parts appears to be an approach to get a hydrocolloid with a higher quality and new functional properties. Therefore, gum tragacanth (GT) was used for these aims. The characterization of alcohol precipitated part of GT were performed in terms of physico-chemical compositions (moisture, ash, protein, color and transparency); rheological behaviors aqueous solutions at different concentration, temperature, $\mathrm{pH}$, presence of salt and sucrose; and structural characterization (FTIR, XRD, DSC and SEM). Applied process has no effect on sucrose its chemical compositions which is important for preserving of progressing application. Clearer solution was obtained for purified sample which was important for sensorial properties of end-product. Rheological experiments indicated that separation insoluble part (bassorin) from GT increased viscosity and improved the stability to the different environmental conditions. FTIR experiment results confirmed that tragacanthin and bassorin is physically mixture not chemically bonded. The improvement of thermal stability of GT was also observed by DSC as a result of bassorin separation. The results suggest that extraction soluble part of GT resulted in higher rheological and structural characteristics which may help to widen its application.
\end{abstract}

Keywords: Characterization, Gum tragacanth, Purification, Rheology, Tragacanthin 


\section{Introduction}

Gum tragacanth (GT) is an anionic polysaccharide produced by drying exudates from the stems and branches of Astragalus species with different compositions. GT has been accepted as GRAS at the level of $0.2-1.3 \%$ since 1961 . GTgrows wildly in South West Asia particularly in Iran and Turkey (Mostafavi, Kadkhodaee, Emadzadeh, \& Koocheki, 2016). GT is obtained into two different types: ribbon and flake. Ribbon type is almost opaque while the flakes are dark (Mohammadifar, Musavi, Kiumarsi, \& Williams, 2006). It is also known as "kitre" in Turkey and uses in production of local ice cream and Turkish dessert, "lokma". The chemical structure is defined as a complex and highly branched polysaccharide consisting of small proportions of protein. Previous studies have reported that gum tragacanth has emulsifying and stabilizing properties in food products due to its high water binding ability and effective surfaceactive properties (Abdolmaleki, Mohammadifar, Mohammadi, Fadavi, \& Meybodi, 2016; A. Kurt, Cengiz, \& Kahyaoglu, 2016; Mostafavi et al., 2016). Pourable, creamy mouth feel and good flavour-release properties of GT were also previously stated by Balaghi, Mohammadifar, Zargaraan, Gavlighi, and Mohammadi (2011) and Levy and Schwarz (1958). The compositions and rheological properties of hydrocolloids are determinants for their usage (Balaghi, Mohammadifar, \& Zargaraan, 2010).

GT consists of two main fractions: a water-soluble (tragacanthin) and an insoluble but water-swellable fraction named bassorin (Balaghi et al., 2011). The ratio of these parts strongly depends on variety which resulted in different flow, structural characteristics and also different functionalities and applications for food systems. In order to acquire GT with a higher quality and independently from species, we aimed to remove insoluble part bassorin from the structure. It is expected that removing bassorin will provide enhancement of interaction between polymer chains and more stable solution against different environmental conditions may be obtained. In addition to stable solutions property, clearer solution is also desired in terms of visual acceptability. Balaghi et al. (2010) also defined the best quality gum tragacanth as tasteless, whitish in color, and translucent in appearance, giving an aqueous solution of high-viscosity, free from sand or bark. Therefore, in the last years, scientific researches have focused on the quality improvement of hydrocolloids by separating the constituents from the main part of gum such as insoluble materials and impurities (Razmkhah, Mohammadifar, Razavi, \& Ale, 2016). The widely applied method consists of following main steps: mixing hydrocolloids in a water with a specific ratio, removing insoluble matter by centrifugation and obtaining specific polysaccharide from the supernatant with different ways such as freeze or spray drying, precipitation with alcohol or separate with the form of complexes by using copper or barium (Abdullah Kurt \& Kahyaoglu, 2017b, 2017c; Razmkhah et al., 2016). Bassorin separated GT had previously obtained from supernatant with different drying methods: spray drying (Koshani, Aminlari, Niakosari, Farahnaky, \& Mesbahi, 2015) and freeze drying (Gavlighi, Meyer, Zaidel, Mohammadifar, \& Mikkelsen, 2013; Mohammadifar et al., 2006). The alcohol precipitation methods were widely used for extraction due to the relatively cost effective, healthier and quick acquiring properties as compared with other mention methods. To the best of our knowledge there are no reports about alcohol precipitation of tragacanthin and their detailed characterization of product.

The easy separation of tragacanthin and bassorin indicated that two polysaccharides are in a physical mixture and not chemically bonded (Balaghi et al., 2010; Koshani et al., 2015). Therefore, to isolate the specific polysaccharide tragacanthin was aimed by applying ethanol precipitation method as a simple and feasible approach in this study. Increased quality will also provide to get higher viscosity at relative low concentrations which are desired characteristics for hydrocolloids. Physicochemical, rheological properties in different $\mathrm{pH}$, salt and sugar concentration and structural variations were evaluated to observe the effect of applied process on potential application of GT.

\section{Materials and Methods}

Gum tragacanth (GT) was kindly donated by Incom (Mersin, Turkey). All chemicals used in this study were of analytical reagent grade. The ethanol, acetone, sodium chloride, calcium chloride, sucrose, lactose were purchased from Sigma Aldrich Chemical Co. (St. Louis, MO, USA). The pH buffer solutions were obtained from Merck (Darmstadt, Germany).

\section{Removing of Bassorin from Gum Tragacanth}

Ten grams of crude gum tragacanth was stirred in $1 \mathrm{~L}$ of distilled water for $1 \mathrm{~h}$ at room temperature, followed by centrifugation $(5000 \mathrm{rpm}, 10 \mathrm{~min})$ to remove basssorin. The supernatants were mixed with absolute ethanol at a ratio of $1: 1$ $(\mathrm{v} / \mathrm{v})$ to precipitate of tragacanthin. The resultant pellets were washed successively with absolute ethanol and acetone and then subsequently dried with a forced air dryer (Mikrotest, Turkey) at $45^{\circ} \mathrm{C}$ overnight. The dried samples were milled, sieved, stored in an airtight bottle at room temperature and coded as BSGT (Bassorin- separated gum tragacanth). 
Physicochemical Analyses

Solubility analyses were performed to determine ratio of soluble to insoluble fraction of crude gum tracagacanth (GT) using the method of Li et al. (2014). $0.1 \mathrm{~g}$ GT was dispersed in $24.90 \mathrm{~g}$ distilled water and the mixture was agitated at room temperature for $1 \mathrm{~h}$. Then mixture was centrifuged for $20 \mathrm{~min}$ at $4500 \mathrm{rpm}$, and $10.00 \mathrm{~g}$ of supernatant was dried to a constant weight at $105^{\circ} \mathrm{C}$. The solubility was calculated as follows:

Solubility $(\%)=\frac{m \times 2.5}{w} \times 100 \%$

where $m$ is the dry matter content of dried supernatant and $\mathrm{w}$ is the total mass of the sample.

The moisture and ash content of samples were analyzed by using gravimetric methods at 105 and $500^{\circ} \mathrm{C}$, respectively. Kjeldahl method was performed for protein determination by the applying the nitrogen conversion factor of 6.25 (Balaghi et al., 2010). Fat content was analyzed by soxhlet method. $\mathrm{pH}$ values of gum solutions $(1 \%)$ were measured with $\mathrm{pH}$ meter (Eutech Instruments, $\mathrm{pH} 700$, Singapore) at room temperature. $L^{*}$ (lightness), $a^{*}$ (redness/greenness) and $b^{*}$ (yellowness/blueness) values of powders were determined by a Minolta Chromameter (CR-400, Minolta Camera Co., Osaka, Japan) to calculate the whiteness index (WI) of the powders as follows (Abdullah Kurt \& Kahyaoglu, 2017a):

$W I=100-\sqrt{\left(100-L^{*}\right)^{2}+\left(a^{*}\right)^{2}+\left(b^{*}\right)^{2}}$

The transparency of $1 \%$ gum solution was evaluated by percent transmittance at $500 \mathrm{~nm}$ against distilled water blank with a Cary 60 UV-visible spectrophotometer (Agilent Technologies, Victoria, Australia) (Kobayashi, Tsujihata, Hibi, \& Tsukamoto, 2002).

\section{Rheological Properties}

Analyses of the rheological properties of gum solutions were performed using rheometer (HAAKE Mars III; Thermo Scientific, Germany) equipped with a Peltier heating system in a cone and plate configuration (diameter: 35 $\mathrm{mm}$, cone angle: $2^{\circ}$, gap size: $0.105 \mathrm{~mm}$ ).

Different concentrations of the samples (1.0 and 2.0\%) were prepared in distilled water with stirring for $1 \mathrm{~h}$ using a magnetic stirrer at room temperature. For each test, samples were allowed to equilibrate for $1 \mathrm{~min}$ at the desired temperature $\left(5,10,25\right.$ and $\left.45^{\circ} \mathrm{C}\right)$. Samples were sheared continuously at a rate ranging from 0 to $300 \mathrm{~s}^{-1}$ in $3 \mathrm{~min}$ for fitting the data to the Ostwald-de Waele model as follows:
$\tau=K . \dot{\gamma}^{n}$

where $\tau$ is the shear stress $(\mathrm{Pa}), \dot{\gamma}$ is the shear rate $\left(\mathrm{s}^{-1}\right), K$ is the consistency coefficient $\left(\mathrm{Pa} . \mathrm{s}^{\mathrm{n}}\right)$, and $n$ is the flow behavior index (dimensionless).

The flow curves were obtained by registering shear stress at shear rates from 0 to $300 \mathrm{~s}-1$ (forward) in $180 \mathrm{~s}$ and down in $180 \mathrm{~s}$ from 300 to $0 \mathrm{~s}-1$ (backward). Thixotropic areas, $A_{t}$ were obtained using data acquisitions software. $A_{t}$ values were calculated using the equation as follows:

$A_{t}=\frac{\left(A_{u p}-A_{\text {down }}\right)}{A_{\text {up }}} \times 100$

Solutions (1\%) were also prepared with different environments: $\mathrm{pH}$ (4.0 and 5.5), salt $\left(\mathrm{NaCl}\right.$ and $\mathrm{CaCI}_{2} ; 10,50$ and $100 \mathrm{mM}$ ) and sugar (sucrose and lactose; 2, 4 and 6\%). Steady flow characteristics of these solutions were determined at $25^{\circ} \mathrm{C}\left(0\right.$ to $300 \mathrm{~s}^{-1}$ in $\left.3 \mathrm{~min}\right)$.

\section{Structural Characterization of GT and BSGT}

Fourier-transform infrared (FTIR) spectroscopy analyses

The FT-IR spectra of samples were recorded on a spectrophotometer (Perkin Elmer, Model Spectrum Two, Ohio, USA) that was fitted with a Miracle Single-Reflection Diamond ATR device in the wavelength range of 4000-650 $\mathrm{cm}^{-1}$ with a spectral resolution of $4 \mathrm{~cm}^{-1}$.

$X$ ray diffraction $(X R D)$ analyses

The XRD patterns of the samples were obtained using a Rigaku SmartLab X-ray diffractometer $(40 \mathrm{kV}, 30 \mathrm{~mA})$ with a scanning rate of $2^{\circ} / \mathrm{min}$ from $5^{\circ}$ to $45^{\circ}$ ( $2 \theta$ range).

\section{Differential scanning calorimetry (DSC) analyses}

The thermal analyses of the samples were performed with a model DSC 4000 (Perkin Elmer, USA). Five milligrams of the sample in an aluminium pan with an empty reference pan were scanned in a nitrogen atmosphere $(20 \mathrm{~mL} / \mathrm{min})$ at a heating rate of $10^{\circ} \mathrm{C} / \mathrm{min}$ in a temperature range of 30 $350^{\circ} \mathrm{C}$.

\section{Scanning electron microscopy (SEM) observation}

The SEM photomicrographs were recorded using a scanning electron microscope (JSM-7001F, JEOL, Japan). The samples were coated with gold-palladium (Quorum SC7620, England) before observation under the microscope. 


\section{Results and Discussion}

\section{Physicochemical Analyses}

The soluble/insoluble ratio is important parameter for the quality of GT which was determined as 2.6 in this study, consistent with the reported study by Gavlighi et al. (2013). Removing bassorin fraction of GT increased this ratio to the highest degree and the flow properties of GT changed significantly. This ratio was reported between $0.5-3.15$ for different species of GM in previous studies (Balaghi et al., 2010). At the end of this purification studies, $35 \%$ of soluble part of GT was obtained by alcohol precipitation method. Ethanol soluble minor fraction, arabinogalactan could be attributed to the decrease in yield which lost with ethanol instead of precipitation. The extraction yield of hydrocolloids varies depending upon the origin and extraction method. Alcohol extraction method of GT is the first attempt and the yield obtained in this study is comparable with the other purified hydrocolloids such as glucomannan and galactomannan (Abdullah Kurt \& Kahyaoglu, 2017b). Spray (Koshani et al., 2015) and freeze (Mohammadifar et al., 2006) drying methods were also conducted to the supernatant phase of GT solutions but there were no information about yield of these methods.

Table 1 summarizes some of the physicochemical properties of GT and BSGT. Total solid, ash and protein contents of GT were not affected from the purification process and obtained results were consistent with the reported study for GT (Mohammadifar et al., 2006) . In similar environmental conditions, different moisture content of hydrocolloid is an evidence of different water polysaccharide interaction and different storage stability (Balaghi et al., 2010). Ash content indicator of mineral content of gum shows variations related to the composition of the soil in which GT grow. During the precipitation of polymeric structure by alcohol, ash constituents were also gained. The presence of protein which plays a pivotal role in gum surface activity makes gum a candidate for emulsification and surface tension reduction. The lower protein content may indicate the purity of hydrocolloids. However purification process did not reduce protein content of GT which could be attribute to the linkages of protein to the molecular structure of hydrocolloids such as gum Arabic (Amid \& Mirhosseini, 2012; Brummer, Cui, \& Wang, 2003; Seyed Mohammad Ali Razavi, Cui, \& Ding, 2016). Small amounts of protein which bounded to the polysaccharide was stated by by Farzi et al. (2011). The result is important for the preservation of this functional property of GT with purification process because previous studies have shown that GT has emulsifying and stabilizing properties, which means that GT could be evaluated for obtaining stable oilin-water emulsions (Abdolmaleki et al., 2016). The samples were not included fat content. Aqueous suspension of samples gave an acidic $\mathrm{pH}$ which found as 5.40 and 5.82 for GT and BSGT, respectively. Acidic nature of gum indicated the presence of uronic acid in polysaccharide structure (Nep \& Conway, 2011). As expected, removing insoluble part had no effect on $\mathrm{pH}$. pH of GT which depends on origins, agricultural history and constituents was reported between 5.25.4 by Teimouri, Abbasi, and Sheikh (2016). Cleared and more stable solutions were desired for gum solutions (Abdullah Kurt \& Kahyaoglu, 2017b). The whiteness index (WI) of powder and transparency of solutions values of GT were presented in Table 1. These parameters of GT increased after purification as a result of removing bassorin and alcohol treatment. The presence of insoluble fragment of GT was responsible for turbidity. Whitish in color and translucent in appearance properties which were the quality parameters of GT were improved by purification study. According to findings of physicochemical analyses it could be said that purification treatment to GT had no effect on chemical constituents but increased the solution properties of GT.

Table 1. Physicochemical properties of crude (GT) and bassorin separated (BSGT) gum tragacanth

\begin{tabular}{ccc}
\hline Properties & GT & BSGT \\
\hline Total Solids (g/100g) & $89.53 \pm 0.67$ & $90.01 \pm 0.01$ \\
Ash $(\mathbf{g} / \mathbf{1 0 0 g})$ & $3.41 \pm 0.02$ & $3.30 \pm 0.03$ \\
Protein $\mathbf{( g / 1 0 0 g )}$ & $1.67 \pm 0.03$ & $1.72 \pm 0.12$ \\
pH & $5.40 \pm 0.00$ & $5.82 \pm 0.02$ \\
Whiteness index (WI) & $81.05 \pm 0.23$ & $90.51 \pm 0.26$ \\
Transparency (\%) & $8.57 \pm 0.00$ & $47.72 \pm 0.00$ \\
\hline
\end{tabular}

$(\mathrm{n}=3) \pm \mathrm{SD}$

\section{Rheological Properties}

\section{Steady-shear flow behavior of gum tragacanth}

The variation in apparent viscosity and Ostwald de Waele model parameters $(K, n)$ of gum tragacanth with the removing bassorin fraction was shown in Table 2 for the different concentration. The viscosity increment with purification was observed for GT. Viscosity is the one of the main parameter to evaluate the quality of polymers (Abdullah Kurt \& Kahyaoglu, 2015). Intermolecular forces and polymer interactions were responsible for the solution viscosity (A. Koocheki, Mortazavi, Shahidi, Razavi, \& Taherian, 2009). Therefore, the interaction between polymer chains and quality of GT increased as a result of removing insoluble part, bassorin. 
Table 2. Apparent viscosity (shear rate: $\left.30 \mathrm{~s}^{-1}\right)$, Ostwald de Waele model parameters $(K, n)$, thixotropic area $\left(\mathrm{A}_{\mathrm{t}}\right)$, activation energy $\left(E_{a}\right)$ of samples.

\begin{tabular}{|c|c|c|c|c|c|c|}
\hline \multirow{2}{*}{ Sample } & Concentration & $\begin{array}{c}\text { Apparent } \\
\text { Viscosity } \\
(\text { Pas })\end{array}$ & $\boldsymbol{K}$ (Pas) & $\boldsymbol{n}$ & $\boldsymbol{A}_{\boldsymbol{t}}(\mathbf{P a} / \mathbf{s})$ & $\begin{array}{c}\boldsymbol{E}_{\boldsymbol{a}} \\
(\mathbf{k J} / \mathbf{m o l})\end{array}$ \\
\cline { 1 - 5 } GT & \multirow{2}{*}{$\mathbf{1 \%}$} & $0.17 \pm 0.00$ & $0.94 \pm 0.04$ & $0.51 \pm 0.01$ & $16.16 \pm 0.21$ & $20.20 \pm 0.26$ \\
\cline { 1 - 5 } BSGT & \multirow{2}{*}{$\mathbf{2 \%}$} & $0.25 \pm 0.00$ & $1.49 \pm 0.02$ & $0.48 \pm 0.00$ & $14.46 \pm 0.62$ & $23.83 \pm 0.23$ \\
\cline { 1 - 7 } GT & $1.02 \pm 0.00$ & $9.82 \pm 0.38$ & $0.33 \pm 0.00$ & $394.55 \pm 0.63$ & $10.22 \pm 0.31$ \\
\cline { 3 - 6 } BSGT & $1.36 \pm 0.02$ & $14.25 \pm 0.12$ & $0.30 \pm 0.00$ & $236.15 \pm 0.49$ & $14.18 \pm 0.25$ \\
\hline
\end{tabular}

$(\mathrm{n}=3) \pm \mathrm{SD}$

The data was fitted by the Ostwald-de Waele model successfully ( $\mathrm{R} 2 \geq 0.99)$. Consistency coefficient $(K)$ is associated with the higher viscosity of the solution since $K$ values indicative of the viscous character of the polymeric system. The larger $K$ values of BSGT were another result of better interaction among the molecules of the polysaccharide. $n$ values were ranging from 0.30 to 0.51 , namely, lower than unity was an evidence of pseudoplasticity. Shear-thinning behavior of GT was previously reported by Farzi et al. (2015). This fact infers that as shear rate increases, the randomly positioned chains of polymer molecules become aligned in the direction of flow, resulting in less interaction among adjacent polymer chains (Arash Koocheki, Taherian, $\&$ Bostan, 2013). Decreased in $n$ value by purification indicated improved pseudoplastic characteristics of GT. In addition, increasing gum concentration resulted in increase of $K$ values whereas $n$ values decreased. Higher content of total solids in solution causes an increase in consistency due to the decrement of intermolecular motion as a result of hydrodynamic forces (Capitani et al., 2015). Similar flow properties of GT was reported previously by Chenlo, Moreira, and Silva (2010).

The differences between upward and downward curves which are the measure of the extent of thixotropy indicate time-dependent rheological characteristics of sample (Seyed M. A. Razavi \& Karazhiyan, 2009). Table 2 shows the calculated hysteresis loop area for GT solutions. Magnitude of the areas decreased slightly at $1 \%$ and markedly at $2 \%$ by removing bassorin which mean that BSGT structure was less damaged from the shear increase and also recovered to the first structure closer than GT (A. Kurt et al., 2016; Roopa \& Bhattacharya, 2009).

The temperature dependency of the consistency index was evaluated by using the Arrhenius model and activation energy $\left(E_{a}\right)$ of purified salep samples were exhibited in Table 2. $E_{a}$ is related to chain flexibility and is an indicator of ability of molecule movement as temperature increases (A.
Koocheki et al., 2009; Arash Koocheki et al., 2013). The higher sensitivity of viscosity to temperature changes implies higher $E_{a}$ value. At all concentrations, $E_{a}$ values increased with the removing bassorin fraction mean that BSGT flow more easily when the temperature is increased due to the higher molecular movement than GT. It could be stated that insoluble fraction (bassorin) of GT had a favorable effect on temperature stability for viscosity. Increasing concentration decreased $E_{a}$ values of GT and BSGT which related to decrease in chain flexibility as a result of decreasing the space for a molecule to flow (A. Koocheki et al., 2009). Similar with reported study by (Mohammadifar et al., 2006) GT exhibited lower $E_{a}$ values than tragacanthin which was the soluble part of GT obtained by freeze drying. The results above suggest that, in order to keep stable viscosity, temperature control is critical for BSGT in food applications.

\section{Effect of pH, Salt and Sugar on Rheological Properties}

$\mathrm{pH}$, salt and sugar effect on consistency coefficient of GT and BSGT shown in Figure1 were evaluated because gums are usually processed under different environmental conditions. As shown in Figure 1A, pH effects were investigated to gain insight into the influence removing insoluble part of GT on the polyelectrolyte nature and solution rheology. Decreasing $\mathrm{pH}$ values decreased $K$ values of GT but increased consistency coefficient of BSGT. The variation of viscosity related to the $\mathrm{pH}$ is attributed to the change in ionization of the groups in molecule such as carboxyl groups and conformational changes in the molecule (Abdullah Kurt \& Kahyaoglu, 2017b). Sharp increment of a gum was attributed to the ionization of its carboxyl groups (MedinaTorres, Brito-De La Fuente, Torrestiana-Sanchez, \& Katthain, 2000; Wu, Ding, Jia, \& He, 2015). The decrease in viscosity at lower $\mathrm{pH}$ values of GT could be a result of insoluble part bassorin that effect the polymer conformation because BSGT exhibited increasing trend when $\mathrm{pH}$ decreased. 


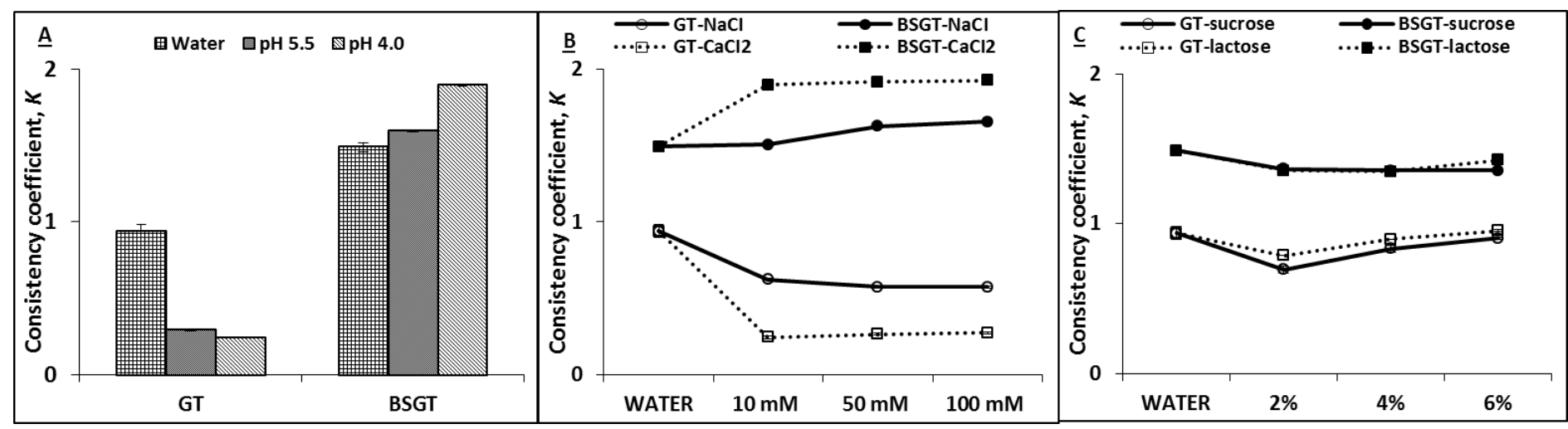

Figure 1. $\mathrm{pH}(\mathrm{A})$, salt-NaCI, $\mathrm{CaCI}_{2}$ (B), sugar-sucrose, lactose (C) effect on consistency coefficent of GT (gum tragacanth) and BSGT (bassorin separated gum tragacanth)

The ionic strength of the solution is an important parameter which has an effect on the networking mechanism of charged biopolymers (Balaghi et al., 2011). The changing consistency of studied gum in different salt and their concentration indicated the polyelectrolyte properties in solution of GT and BSGT (Abdullah Kurt \& Kahyaoglu, 2017a; Medina-Torres et al., 2000). As shown in Figure 1B, both salt types $\left(\mathrm{NaCI}\right.$ and $\left.\mathrm{CaCI}_{2}\right)$ addition decreased the consistency coefficient of GT similar with the reported studies (Balaghi et al., 2011; Mohammadifar et al., 2006) but the salts led to an increase in this property of BSGT which exhibited rapid reduction and increment, respectively for $10 \mathrm{mM}$ salt concentration. At increasing concentration, viscosity values of both gum exhibited stable trends. Comparatively, the viscosity variation is more sensitive to $\mathrm{CaCI}_{2}$ than $\mathrm{NaCI}$ which consistent with the reported study for tara gum due to the stronger effect of divalent salt $\mathrm{CaCI}_{2}$ than $\mathrm{NaCI}$ (Capitani et al., 2015). Gum tragacanth is known as a branched, heterogeneous, and anionic carbohydrate (Balaghi et al., 2010). Negatively charged groups causes expansions of molecules in solution which lead to the higher viscosity if the solutions are not included any salts. However addition positive ions are resulted in different flow behavior. These observations were attributed to the forming junctions and reinforcing interaction between the molecules with the aid of ions for BSGT which resulted viscosity increment (Farahnaky, Shanesazzadeh, Mesbahi, \& Majzoobi, 2013). On the other hand, insoluble part could be exhibited hurdle effect between the molecules which lost its expansion as a result of added positive ions, resulted viscosity decrement.

Addition of $2 \%$ sucrose and lactose resulted sharply decrement in $K$ values of both gums (Figure1C). Increase in concentration provided an increase in viscosities which resemble its water condition for GT. However increasing sugar concentration after $2 \%$ did not change solution viscosity of BSGT. Extending of polymer molecules was reduced by sugar due to the decrement of available water for gum hydration which leads to the viscosity drop (Wu et al., 2015). Another explanation is that decrease in the size of polymers as a result of the decrease in the macromolecular association at the presence of sugar, which was observed for locust bean gum and purified salep glucomannan (Abdullah Kurt \& Kahyaoglu, 2017a; Richardson, Willmer, \& Foster, 1998). It can be concluded that the viscosity of BSGT in different conditions showed higher values than GT indicated that removing insoluble part fraction of GT improved gum quality (i), provided stability (ii) and decreased gum requirement to obtained desired viscosities (iii).

\section{Structural Characterization of GT and BSGT}

\section{FTIR spectroscopy analysis}

The FTIR spectrum of GT and BSGT was shown in Fig. 2A to gain further insights effect of removing bassorin on the structure and bonding in the polymer. Broad band around $3330 \mathrm{~cm}^{-1}$ was due to stretching vibrations of $-\mathrm{OH}$ group related to free, inter, and intra-molecular bound hydroxyl groups and the band at $2940 \mathrm{~cm}^{-1}$ is an indicator of $\mathrm{C}-\mathrm{H}$ stretching (Antoniou, Liu, Majeed, \& Zhong, 2015; Abdullah Kurt \& Kahyaoglu, 2015). The peaks at $1744 \mathrm{~cm}^{-1}$ could be assigned to carbonyl stretching vibrations $(\mathrm{C}=\mathrm{O}$ stretching of $-\mathrm{COOH}$ ) (Zarekhalili, Bahrami, Ranjbar-Mohammadi, \& Milan, 2017).The intensity of the band in the region $1623-1644 \mathrm{~cm}^{-1}$ attributed to the presence of protein in the structure (Chua et al., 2012). The observed different rheological behavior of GT and BSGT at different salt and $\mathrm{pH}$ environment was the evidence of the presence of uronic acid in structure which related to wavenumber about $1400 \mathrm{~cm}^{-1}$ (Abdullah Kurt \& Kahyaoglu, 2017c; Seyed Mohammad Ali Razavi, Cui, Guo, \& Ding, 2014). The bands at 1245, 
1079 and $1024 \mathrm{~cm}^{-1}$ were related to stretching vibrations of $\mathrm{C}-\mathrm{O}$ in polyols, ethers and alcohol groups, respectively (Zarekhalili et al., 2017). The peaks between 800 and 1200 $\mathrm{cm}^{-1}$ were pointed out the finger print region for carbohydrates (Nep \& Conway, 2011). FTIR spectra of bassorin separated gum tragacanth did not show significant difference in terms of intensity, the position of absorption bands, no shift of peaks and new peak occurrence in comparison to GT, indicating applied process to the GT to remove bassorin preserved the chemical structure of gum. Therefore, FTIR experiment results confirmed that two different fractions of GT, tragacanthin and bassorin are mixture in physical form instead of chemical (Farzi et al., 2015).

\section{$X R D$ analyses}

XRD experiments were conducted to determine crystallinity of GT and effect on purification on that (Figure 2B). XRD pattern of samples showed a broad peak (at $2 \theta$ about $20^{\circ}$ ) due to amorphous nature of GT which consistent with the reported studies (Singh \& Sharma, 2017; Singh, Varshney, Francis, \& Rajneesh, 2016). Bassorin separation from GT resulted in lower broad peak intensity with an amorphous nature. Amorphous materials exhibit more hygroscopic characteristics than crystalline structure due to the absorption properties which caused to increase of exposed absorption sites (Abdullah Kurt \& Kahyaoglu, 2017a; Xiao et al., 2015). Therefore GT and BSGT are soluble in water even at room temperature.

\section{DSC analyses}

DSC thermograms of GT and BSGT were shown in Figure $2 \mathrm{C}$. There were two main peaks. The first transition endothermic peak could be ascribed to be due to vaporization of moisture or bound water present in the samples (Abdullah Kurt \& Kahyaoglu, 2017c). The lack of melting endothermic peak also confirmed the amorphous nature of samples. The second peak exhibited exothermic transition which was the main decomposition of molecular chain (Li et al., 2014). Applied process made this peak narrower which indicated relative purity of BSGT (Pawar \& Lalitha, 2014). The onset of the exothermic peak temperature $\left(\mathrm{T}_{\text {onset }}\right)$ and the maximum temperature $\left(\mathrm{T}_{\max }\right)$ of GT increased from 237.72 to $251.98{ }^{\circ} \mathrm{C}$ and from 258.68 to $263.43^{\circ} \mathrm{C}$, respectively, as a result of bassorin separation, which showed the improvement of thermal stability due to the enhancement of hydrogen bonding interaction in the structure of BSGT. The decomposition of molecular chain reported between 250 and $280^{\circ} \mathrm{C}$ for GT (Zarekhalili et al., 2017) which agree with our results. The higher decomposition temperature of BSGT can be correlated with its stable structure of BSGT during various food processes that involves higher temperatures.

\section{SEM analyses}

SEM micrographs of samples were presented in Figure 4D at $500 \times$ magnification. The shape of granule and surface morphology variations as a consequence of ethanol treatment to GT were observed with this study. Firstly, shape of granule exhibited good agreement with reported study for GT (Alijani, Balaghi, \& Mohammadifar, 2011). The images indicated that applied process preserved shape of granules and no destructive effect was observed as expected. The result was another confirmation of coexisting bassorin and tragacanthin in physically. The smoother surface of granules appeared with ethanol treatment which is consistent with the study (Abdullah Kurt \& Kahyaoglu, 2015) and responsible for the higher whiteness of BSGT.

\section{Conclusions}

The bassorin separation from gum tragacanth had no effect on its chemical structure but solution transparency was improved. The viscosities of bassorin separated sample solution are higher than those of gum tragacanth solutions at the same gum concentration, $\mathrm{pH}$, salt and sugar conditions. The more stable gum solution was obtained by purification at lower $\mathrm{pH}$ and higher salt concentrations. Thermal stability of gum tragacanth was also improved with this process. The results obtained in this study could be useful for widening the application of gum tragacanth in the food, chemical and polymer industries due to the better rheological and structural characteristics. 

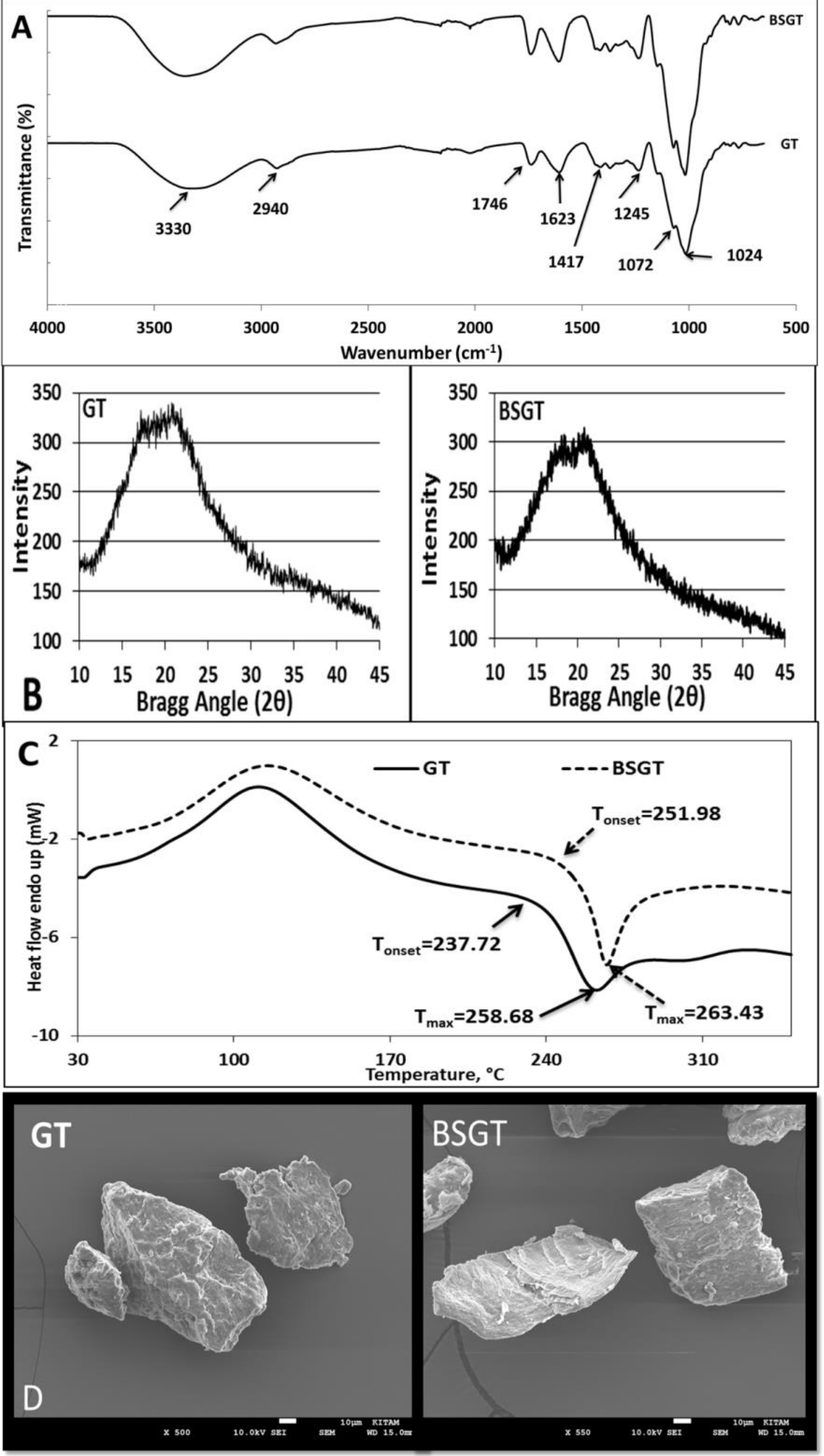

Figure 2. FTIR spectra (A), XRD patterns (B), DSC thermograms (C) and SEM images (D) of GT (gum tragacanth) and BSGT (bassorin separated gum tragacanth) 


\section{References}

Abdolmaleki, K., Mohammadifar, M.A., Mohammadi, R., Fadavi, G., Meybodi, N.M. (2016). The effect of $\mathrm{pH}$ and salt on the stability and physicochemical properties of oil-in-water emulsions prepared with gum tragacanth. Carbohydrate Polymers, 140, 342-348.

Alijani, S., Balaghi, S., Mohammadifar, M.A. (2011). Effect of gamma irradiation on rheological properties of polysaccharides exuded by A. fluccosus and A. gossypinus. International Journal of Biological Macromolecules, 49(4), 471-479.

Amid, B.T., Mirhosseini, H. (2012). Emulsifying Activity, Particle Uniformity and Rheological Properties of a Natural Polysaccharide-Protein Biopolymer from Durian Seed. Food Biophysics, 7(4), 317-328.

Antoniou, J., Liu, F., Majeed, H., Zhong, F. (2015). Characterization of tara gum edible films incorporated with bulk chitosan and chitosan nanoparticles: A comparative study. Food Hydrocolloids, 44, 309-319.

Balaghi, S., Mohammadifar, M.A., Zargaraan, A. (2010). Physicochemical and Rheological Characterization of Gum Tragacanth Exudates from Six Species of Iranian Astragalus. Food Biophysics, 5(1), 59-71.

Balaghi, S., Mohammadifar, M.A., Zargaraan, A., Gavlighi, H.A., Mohammadi, M. (2011). Compositional analysis and rheological characterization of gum tragacanth exudates from six species of Iranian Astragalus. Food Hydrocolloids, 25(7), 1775-1784.

Brummer, Y., Cui, W., Wang, Q. (2003). Extraction, purification and physicochemical characterization of fenugreek gum. Food Hydrocolloids, 17(3), 229-236.

Capitani, M.I., Corzo-Rios, L.J., Chel-Guerrero, L.A., Betancur-Ancona, D.A., Nolasco, S.M., Tomás, M.C. (2015). Rheological properties of aqueous dispersions of chia (Salvia hispanica L.) mucilage. Journal of Food Engineering, 149, 70-77.

Chenlo, F., Moreira, R., Silva, C. (2010). Rheological properties of aqueous dispersions of tragacanth and guar gums at different concentrations. Journal of Texture Studies, 41(3), 396-415.
Chua, M., Chan, K., Hocking, T.J., Williams, P.A., Perry, C.J., Baldwin, T. C. (2012). Methodologies for the extraction and analysis of konjac glucomannan from corms of Amorphophallus konjac K. Koch. Carbohydrate Polymers, 87(3), 2202-2210.

Farahnaky, A., Shanesazzadeh, E., Mesbahi, G., Majzoobi, M. (2013). Effect of various salts and $\mathrm{pH}$ condition on rheological properties of Salvia macrosiphon hydrocolloid solutions. Journal of Food Engineering, 116(4), 782-788.

Farzi, M., Saffari, M.M., Emam-Djomeh, Z., Mohammadifar, M.A. (2011). Effect of ultrasonic treatment on the rheological properties and particle size of gum tragacanth dispersions from different species. International Journal of Food Science and Technology, 46(4), 849854.

Farzi, M., Yarmand, M.S., Safari, M., Emam-Djomeh, Z., Mohammadifar, M.A. (2015). Gum tragacanth dispersions: Particle size and rheological properties affected by high-shear homogenization. International Journal of Biological Macromolecules, 79, 433-439.

Gavlighi, H.A., Meyer, A.S., Zaidel, D.N.A., Mohammadifar, M.A., Mikkelsen, J.D. (2013). Stabilization of emulsions by gum tragacanth (Astragalus spp.) correlates to the galacturonic acid content and methoxylation degree of the gum. Food Hydrocolloids, 31(1), 514.

Kobayashi, S., Tsujihata, S., Hibi, N., Tsukamoto, Y. (2002). Preparation and rheological characterization of carboxymethyl konjac glucomannan. Food Hydrocolloids, 16(4), 289-294.

Koocheki, A., Mortazavi, S.A., Shahidi, F., Razavi, S.M.A., Taherian, A.R. (2009). Rheological properties of mucilage extracted from Alyssum homolocarpum seed as a new source of thickening agent. Journal of Food Engineering, 91(3), 490-496.

Koocheki, A., Taherian, A.R., Bostan, A. (2013). Studies on the steady shear flow behavior and functional properties of Lepidium perfoliatum seed gum. Food Research International, 50(1), 446-456. 
Koshani, R., Aminlari, M., Niakosari, M., Farahnaky, A., Mesbahi, G. (2015). Production and properties of tragacanthin-conjugated lysozyme as a new multifunctional biopolymer. Food Hydrocolloids, 47, 69-78.

Kurt, A., Cengiz, A., Kahyaoglu, T. (2016). The effect of gum tragacanth on the rheological properties of salep based ice cream mix. Carbohydrate Polymers, 143, 116-123.

Kurt, A., Kahyaoglu, T. (2015). Rheological properties and structural characterization of salep improved by ethanol treatment. Carbohydrate Polymers, 133, 654-661.

Kurt, A., Kahyaoglu, T. (2017a). Gelation and structural characteristics of deacetylated salep glucomannan. Food Hydrocolloids, 69, 255-263.

Kurt, A., Kahyaoglu, T. (2017b). Purification of glucomannan from salep: Part 1. Detailed rheological characteristics. Carbohydrate Polymers, 168, 138-146.

Kurt, A., Kahyaoglu, T. (2017c). Purification of glucomannan from salep: Part 2. Structural characterization. Carbohydrate Polymers, 169, 406-416.

Levy, G., Schwarz, T. W. (1958). Tragacanth Solutions I. Journal of the American Pharmaceutical Association (Scientific ed.), 47(6), 451-454.

Li, J., Ye, T., Wu, X., Chen, J., Wang, S., Lin, L., Li, B. (2014). Preparation and characterization of heterogeneous deacetylated konjac glucomannan. Food Hydrocolloids, 40, 9-15.

Medina-Torres, L., Brito-De La Fuente, E., TorrestianaSanchez, B., Katthain, R. (2000). Rheological properties of the mucilage gum (Opuntia ficus indica). Food Hydrocolloids, 14(5), 417-424.

Mohammadifar, M.A., Musavi, S.M., Kiumarsi, A., Williams, P.A. (2006). Solution properties of targacanthin (water-soluble part of gum tragacanth exudate from Astragalus gossypinus). International Journal of Biological Macromolecules, 38(1), 31-39.

Mostafavi, F.S., Kadkhodaee, R., Emadzadeh, B., Koocheki, A. (2016). Preparation and characterization of tragacanth-locust bean gum edible blend films. Carbohydrate Polymers, 139, 20-27.
Nep, E.I., Conway, B.R. (2011). Physicochemical characterization of grewia polysaccharide gum: Effect of drying method. Carbohydrate Polymers, 84(1), 446-453.

Pawar, H.A., Lalitha, K.G. (2014). Isolation, purification and characterization of galactomannans as an excipient from Senna tora seeds. International Journal of Biological Macromolecules, 65, 167-175.

Razavi, S.M.A., Cui, S.W., Ding, H. (2016). Structural and physicochemical characteristics of a novel water-soluble gum from Lallemantia royleana seed. International Journal of Biological Macromolecules, 83, 142-151.

Razavi, S.M.A., Cui, S.W., Guo, Q., Ding, H. (2014). Some physicochemical properties of sage (Salvia macrosiphon) seed gum. Food Hydrocolloids, 35, 453462.

Razavi, S.M.A., Karazhiyan, H. (2009). Flow properties and thixotropy of selected hydrocolloids: Experimental and modeling studies. Food Hydrocolloids, 23(3), 908-912.

Razmkhah, S., Mohammadifar, M.A., Razavi, S.M.A., Ale, M.T. (2016). Purification of cress seed (Lepidium sativum) gum: Physicochemical characterization and functional properties. Carbohydrate Polymers, 141, 166-174.

Richardson, P.H., Willmer, J., Foster, T.J. (1998). Dilute solution properties of guar and locust bean gum in sucrose solutions. Food Hydrocolloids, 12(3), 339-348.

Roopa, B.S., Bhattacharya, S. (2009). Characterisation and modelling of time-independent and time-dependent flow behaviour of sodium alginate dispersions. International Journal of Food Science and Technology, 44(12), 2583-2589.

Singh, B., Sharma, V. (2017). Crosslinking of poly(vinylpyrrolidone)/acrylic acid with tragacanth gum for hydrogels formation for use in drug delivery applications. Carbohydrate Polymers, 157, 185-195.

Singh, B., Varshney, L., Francis, S., Rajneesh. (2016). Designing tragacanth gum based sterile hydrogel by radiation method for use in drug delivery and wound dressing applications. International Journal of Biological Macromolecules, 88, 586-602. 
Teimouri, S., Abbasi, S., Sheikh, N. (2016). Effects of gamma irradiation on some physicochemical and rheological properties of Persian gum and gum tragacanth. Food Hydrocolloids, 59, 9-16.

Wu, Y., Ding, W., Jia, L., He, Q. (2015). The rheological properties of tara gum (Caesalpinia spinosa). Food Chemistry, 168, 366-371.
Xiao, M., Dai, S., Wang, L., Ni, X., Yan, W., Fang, Y., Corke H., Jiang F. (2015). Carboxymethyl modification of konjac glucomannan affects water binding properties. Carbohydrate Polymers, 130, 1-8.

Zarekhalili, Z., Bahrami, S.H., Ranjbar-Mohammadi, M., Milan, P.B. (2017). Fabrication and characterization of PVA/Gum tragacanth/PCL hybrid nanofibrous scaffolds for skin substitutes. International Journal of Biological Macromolecules Part A, 94, 679-690. 